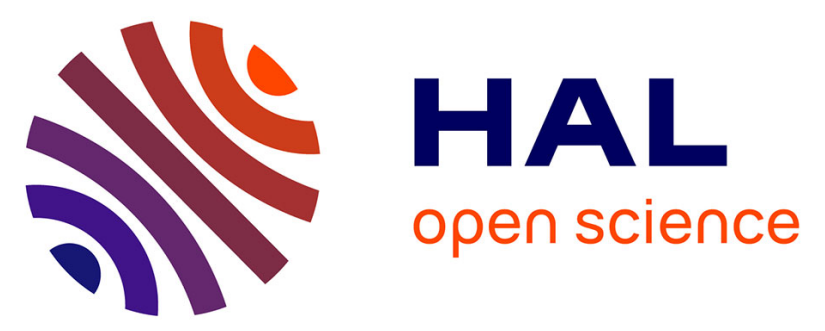

\title{
Size Characterization of Glutathione-Protected Gold Nanoclusters in the Solid, Liquid and Gas Phases
}

\author{
Antonin Soleilhac, Franck Bertorelle, Clothilde Comby-Zerbino, Fabien
}

Chirot, Nathalie Calin, Philippe Dugourd, Rodolphe Antoine

\section{To cite this version:}

Antonin Soleilhac, Franck Bertorelle, Clothilde Comby-Zerbino, Fabien Chirot, Nathalie Calin, et al.. Size Characterization of Glutathione-Protected Gold Nanoclusters in the Solid, Liquid and Gas Phases. Journal of Physical Chemistry C, 2017, 121 (49), pp.27733 - 27740. 10.1021/acs.jpcc.7b09500 . hal01679519

\section{HAL Id: hal-01679519 \\ https://hal.science/hal-01679519}

Submitted on 1 Nov 2020

HAL is a multi-disciplinary open access archive for the deposit and dissemination of scientific research documents, whether they are published or not. The documents may come from teaching and research institutions in France or abroad, or from public or private research centers.
L'archive ouverte pluridisciplinaire HAL, est destinée au dépôt et à la diffusion de documents scientifiques de niveau recherche, publiés ou non, émanant des établissements d'enseignement et de recherche français ou étrangers, des laboratoires publics ou privés. 


\title{
On the Size Characterization of Glutathione-
}

\section{Protected Gold Nanoclusters in the Solid, Liquid and}

\section{Gas Phases}

Antonin Soleilhac, ${ }^{1}$ Franck Bertorelle, ${ }^{1}$ Clothilde Comby-Zerbino, ${ }^{1}$ Fabien Chirot, ${ }^{2}$ Nathalie

Calin, ${ }^{3}$ Philippe Dugourd ${ }^{l}$ and Rodolphe Antoine ${ }^{l, *}$

${ }^{1}$ Univ Lyon, Université Claude Bernard Lyon 1, CNRS, Institut Lumière Matière, UMR 5306, F-69622 Lyon, France

${ }^{2}$ Univ Lyon, Université Claude Bernard Lyon 1, ENS de Lyon, CNRS, Institut des Sciences Analytiques, UMR 5280, 5 rue de la Doua, F-69100 Villeurbanne, France

${ }^{3}$ Laboratoire de Chimie UMR 5182, Ecole Normale Supérieure de Lyon, CNRS Université Lyon 1, 46 allée d'Italie, Lyon 69007, France

\begin{abstract}
Monolayer-protected metal nanoclusters belonging to the size regime between two and a few hundred atoms represent unique building blocks for new materials. Due to the extremely tiny size of such clusters (smaller than $\sim 3 \mathrm{~nm}$ in diameter) and the stabilizing layer which varies in size and composition, traditional techniques used for characterizing the size of large metal nanoparticles are not very reliable. In this work, we compare solid, liquid and gas phase size characterization methods for selected glutathione (SG)-protected gold clusters (namely $\mathrm{Au}_{15}(\mathrm{SG})_{13}, \mathrm{Au}_{18}(\mathrm{SG})_{14}$ and $\left.\mathrm{Au}_{25}(\mathrm{SG})_{18}\right)$. We also investigate the effect of nanocluster ion-pairing with bulky counterions on their size characterization. We show that X-ray powder diffraction, ion mobility mass spectrometry and time-resolved fluorescence anisotropy can be a useful addition to tools available for ultra-small nanoparticle characterization.
\end{abstract}




\section{INTRODUCTION.}

"Size matters" has become the catchphrase of nanoscience because the nano-world is all about the question of size. Since the size of a nanoparticle plays a crucial role in its properties, sizing is a primary task in characterizing the properties of nanoparticles. In this context, electron-based microscopy is considered the gold standard for nanoparticle characterization. ${ }^{1-2}$ Below a size of 1$3 \mathrm{~nm}$, nanoparticles, more often called nanoclusters, enter the range where "each atom counts", and their properties are often dominated by quantum effects. ${ }^{3-5}$ Therefore, controlling the composition and structure of nanoclusters with atomic precision has now become a major and challenging goal in the nanotechnology community. Recently, this goal has been partially reached with monolayer-protected quantum sized gold clusters ${ }^{6-8}$ One commonly studied class of clusters is thiolate protected gold clusters, with the molecular formula $\mathrm{Au}_{\mathrm{x}} \mathrm{SR}_{\mathrm{y}}$. Many variations of these clusters have been synthesized by tuning the atom counts for the metal (x), and by using different types of coordinating SR thiols. For example, the most commonly synthesized/studied cluster has the formula $\mathrm{Au}_{25} \mathrm{SR}_{18}\left(25\right.$ gold atoms and 18 thiol ligands). ${ }^{9-10}$

As stated by Rongchao Jin in his last review, ${ }^{6} \mathrm{X}$-ray structures are the "holy grail" of nanocluster research. Unraveling the total structure of gold nanoclusters is of paramount importance for their in-depth characterization. ${ }^{11-13}$ Unfortunately, the use of X-ray crystallography is problematic, because sample crystallization requires extremely high purity and stability. The difficulty of extracting quantitative information from the diffraction patterns of nanosized and poorly crystallized compounds is the reason why X-ray Powder Diffraction (XRPD) techniques were mostly used to validate the structural and microstructural features obtained by other techniques such as electron microscopy. ${ }^{14}$ Although conventional TEM is a powerful technique for determining particle sizes with a diameter larger than $1 \mathrm{~nm}$, the contrast becomes very weak for 
very small particles $(\sim 1 \mathrm{~nm})$ and hence such gold nanoclusters might not be detected. ${ }^{5}$ Also, when the sample is prepared from solutions, the clusters may form densely packed aggregates, preventing accurate size determination.

Diffusion ordered NMR spectroscopy was used as an analytical tool to estimate the size of thiolstabilized gold nanoclusters in solution. ${ }^{15}$ Also popularly employed method is analytical ultracentrifugation, to obtain hydrodynamic radius via the determination of the diffusion constant. ${ }^{16-17}$ Among other conventionally used methods, gel-electrophoresis (PAGE) can be calibrated to provide a measure of solution-phase size (analogous to gas-phase electrophoretic mobility), but that analytical liquid chromatography (retention times) are usually not so amenable to conversion into a hydrodynamic radius. ${ }^{18}$ Cryo-TEM single particle reconstruction, ${ }^{19}$ electron diffraction crystallography ${ }^{20}$ have been recently used to gain insights at various levels. Also, mass spectrometry (MS) based methods and in particular electrospray MS (ESI-MS) have proven to be much more user-friendly and have become standard characterization methods. ${ }^{21-23}$ More recently, ion mobility mass spectrometry (IM-MS) has proved to be a useful complement to MS due to its ability to separate ions ${ }^{24}$ based on their size, shape, charge and mass-to-charge ratios, as shown with $\mathrm{Au}_{25} \mathrm{SR}_{18}$ by Dass and coworkers. ${ }^{25}$ In this work, we take this coupling further by reporting IM-derived collision cross-sections (CCS) for selected gas phase glutathione-protected gold cluster ions. CCS values provide a measure of the shape and structure of nanoclusters together with exact mass determination using MS.

Since such nanoclusters present stable suspensions in solution, dynamic light scattering (DLS) could be used to characterize them in a solution. However, in the case of such small clusters, evaluating size becomes difficult due to the lack of sensitivity of DLS techniques. Fluorescence anisotropy analysis may be an alternative for nanoparticle sizing as it allows determining the 
rotational diffusion coefficient ${ }^{26}$. Fluorescence anisotropy measurements have been used to evaluate the size modification of bovine serum albumin (BSA)- $\mathrm{Au}_{25}$ clusters as the $\mathrm{pH}$ is reduced from 7 to 3.1. ${ }^{27}$ Indeed, quantum sized gold nanoclusters have been found to present considerable fluorescence anisotropy. ${ }^{28}$ However, although they present emissive properties, ${ }^{29-30}$ quantum yields usually range between $0.01 \%$ to $0.4 \%$, which may make the above-mentioned technique more difficult to implement. Such low quantum yields may be due to the "floppiness" of the surface ligand shell, favoring non-radiative decay. ${ }^{31}$ To achieve higher rigidity of the gold-ligand shell, gold clusters can be bound to bulky tetraoctylammonium cations, resulting in enhanced quantum yield luminescence at room temperature. ${ }^{32}$ In the following, we show that selected luminescent glutathione-protected gold clusters in suspension can be sized based on fluorescence anisotropy measurements.

In this paper, we present an inter-comparison of solid, liquid and gas phase methods used for "sizing" selected glutathione-protected gold clusters, and show how the different measurements can be used to give complementary results for the 1-3 nm size range. Multiple size determination in this size range is new and paves the way for synthesis quality control, a prerequisite for future applications.

\section{EXPERIMENTAL SECTION.}

Materials: Hydrogen tetrachloroaurate(III) hydrate $\left(\mathrm{HAuCl}_{4}, 3 \mathrm{H}_{2} \mathrm{O}\right)$ was purchased from Alfa Aesar. L-glutathione reduced (GSH), triethylammine, tributylamine were purchased from Carl Roth. Tetramethylammonium borohydride, tetrabutylammonium borohydride, tetrabutylammonium hydroxide were purchased from Sigma-Aldrich. Ultrapure water with a 
resistance of 18.2 M $\Omega$ was used throughout the experiment. Methanol, ethanol, glycerol (analytical grade) were purchased from Carl Roth.

$A u_{15}(S G)_{13}$ cluster synthesis: $\mathrm{Au}_{15}(\mathrm{SG})_{13}$ was synthesized as reported by Russier-Antoine et al. ${ }^{33}$ $A u_{18}(S G)_{14}$ and $A u_{25}(S G)_{18}$ cluster synthesis: The synthesis of size focusing $\operatorname{Aux}(\mathrm{SG}) \mathrm{y}$ cluster is based on a kinetic and thermodynamic control. Kinetic control is directed by factors such as temperature, solvent, reducing agent and reactant ratio while thermodynamic control is mainly directed by thiol etching. In our case, composition of the solvent (the ratio methanol/water/tributylamine or triethylamine), temperature and quantities of reducer (in multiple steps) are the principals keys factors that leads to $\mathrm{Au}_{18}(\mathrm{SG})_{14}$ and $\mathrm{Au}_{25}(\mathrm{SG})_{18} \cdot A_{18}(S G)_{14}$ cluster: $390 \mathrm{mg}$ of glutathione (GSH) is dissolved in $45 \mathrm{~mL}$ of methanol, $8 \mathrm{ml}$ of tributylamin and $4.5 \mathrm{~mL}$ of water. Then $2 \mathrm{ml}$ of gold ( $200 \mathrm{mg}$ of $\mathrm{HAuCl}_{4}, 3 \mathrm{H}_{2} \mathrm{O}$ in water) and $20 \mathrm{ml}$ of diethyl ether are added. The solution is mixed in an ice bath for $15 \mathrm{~min} .80 \mathrm{mg}$ of tetrabutylammonium borohydride powder is added in two parts $(2 \times 40 \mathrm{mg})$ spaced by $45 \mathrm{~min}$, under strong agitation. 45 min later, the solution has turned yellow and is removed from the ice bath. Then $200 \mathrm{mg}$ of tetramethylammonium borohydride is added and the solution is stirred for 3 hours. $A u_{25}(S G)_{18}$ : $234 \mathrm{mg}$ of glutathione (GSH) is dissolved in $35 \mathrm{ml}$ of methanol, $2 \mathrm{ml}$ of tributylamine and $2 \mathrm{ml}$ of triethylamine. Then $100 \mathrm{mg}$ of $\mathrm{HAuCl}_{4}, 3 \mathrm{H}_{2} \mathrm{O}$ dissolved in $10 \mathrm{ml}$ of water is added and the solution is stirred for 3 hours at $45^{\circ} \mathrm{C}$. The solution is then cooled to ambient temperature and $50 \mathrm{mg}$ of tetramethylammonium borohydride is added under strong stirring. 1 hour later another $25 \mathrm{mg}$ of borohydride is added and the solution is stirred for 3 hours. The solution is finally left undisturbed overnight before purification. 
Purification step: The precipitation of NCs is induced by adding $1 \mathrm{ml}$ of $\mathrm{NaOH}(1 \mathrm{M})$ (or $1 \mathrm{ml}$ of $\mathrm{NH}_{4} \mathrm{OH} 10 \%$ ). The unwanted products are removed with cycles of dissolution/precipitation. The powder is dissolved in a minimum volume of $\mathrm{H}_{2} \mathrm{O} / \mathrm{NH}_{4} \mathrm{OH}$ and precipitated with $\mathrm{MeOH}$. After centrifugation, the powder is dissolved again in $10 \mathrm{ml}$ of water. Then $2 \mathrm{ml}$ of glacial acetic acid is added and the solution is left undisturbed for 1 hour before being centrifuged. The supernatant is collected and then precipitated with $\mathrm{MeOH}$ before being dried under vacuum.

AuNCs(TBA) preparation:AuNCs $(50 \mathrm{mg})$ are dissolved in $1 \mathrm{ml}$ of water with $200 \mathrm{mg}$ of tetrabutylammonium hydroxide ( $\left.\mathrm{TBAOH}, 30 \mathrm{H}_{2} \mathrm{O}\right)$. This corresponds to one glutathione molecule for $3 \mathrm{Bu} 4 \mathrm{~N}$ molecules. In our experiments, due to basic conditions, we expect that all carboxylic groups of glutathione are deprotonated. However, some steric hindrance might avoid a full ionpairing formation with all the $\mathrm{COO}^{-}$surface groups. Then $3 \mathrm{ml}$ of methanol is added and TBAAuNCS is precipitated with diethyl ether.

Electrospray Ionization Mass Spectrometry (ESI-MS). ESI-MS was performed on a commercial quadrupole time-of-flight (micro-qTOF, Bruker-Daltonics, Bremen, Germany, mass resolution $10000) .{ }^{34}$ The samples were prepared to a final concentration of approximately $50 \mu \mathrm{M}$ in water and analyzed in negative ion mode. External calibration was carried out with a set of synthetic peptides. MS spectra of the as-synthesized in $\mathrm{Au}_{15}(\mathrm{SG})_{13}, \mathrm{Au}_{18}(\mathrm{SG})_{14}$ and $\mathrm{Au}_{25}(\mathrm{SG})_{18}$ NCS in aqueous solutions are presented in fig. S1 in supporting information.

X-ray Powder Diffraction (XRD) Measurements. XRD experiments were carried out using a PANalytical EMPYREAN diffractometer with a PIXcel 3D detector using $\mathrm{Cu}-\mathrm{K} \alpha$ monochromatic radiation $(\lambda=0.154184 \mathrm{~nm})$ and a high-resolution theta-theta goniometer. XRD patterns were 
measured between 12 and $80^{\circ}$ in $2 \theta$ at room temperature (with a step size of $0.026^{\circ}$ and a time per step of $600 \mathrm{~s})$.

The first peak at small $2 \theta$ corresponds to the inter-particle diffraction pattern and is associated with the center-to-center particle distance $d_{\text {center-center }}$ given by the Bragg's law:

$$
d_{\text {center-center }}=\frac{n \lambda}{2 \times \sin (\theta)}
$$

Where $n$ is the diffraction order, $\lambda$ the excitation wavelength, and $\theta$ the diffraction angle. The high peak at $2 \theta \sim 37^{\circ}$ corresponds to the diffraction of the NC gold core. The gold core diameter $d_{\text {core }}$ is given by its Full Width at Half Maximum (FWHM) and its diffraction angle $\theta$ by DebyeScherrer' law:

$$
d_{\text {core }}=\frac{0.9 \lambda}{F W H \times \cos (\theta)}
$$

Ion Mobility Mass Spectrometry. Ion mobility measurements were performed on a homemade drift tube time-of-flight instrument described in detail elsewhere. ${ }^{35}$ The nanocluster samples were prepared at a final concentration of approximately $30 \mu \mathrm{M}$ in water. The samples are analyzed directly in negative electrospray mode. Electrosprayed metal cluster ions are first trapped in a dualstage ion funnel assembly and ion packets are periodically injected in a $79 \mathrm{~cm}$ drift tube across which they travel driven by a uniform electric field $E$ through a buffer gas (He) at a pressure of 4 Torr and maintained at room temperature. The metal cluster ions are finally conveyed by a series of ion optics to a time-of-flight mass spectrometer. Each ion detected can then be associated with an arrival time (in the IM dimension) and a mass-to-charge ratio. Under the experimental 
conditions the ions travel across the drift tube at a constant speed $v_{D}$, and their drift time $t_{D}$ through the tube is linked to this field through:

$$
t_{D}=\frac{L}{v_{D}}=\frac{L}{K E}
$$

where $K$ defines the ion mobility. $K$ is inversely proportional to the orientationally averaged diffusion cross-section CCS $: 35$

$$
K=\frac{3}{16} \times \frac{z e}{N} \times\left(\frac{1}{m}+\frac{1}{M}\right)^{1 / 2} \times\left(\frac{2 \pi}{k T}\right)^{1 / 2} \times \frac{1}{\mathrm{CCS}}
$$

where $z e$ is the charge of the ion, $N$ is the buffer gas density, $k$ is the Boltzmann constant, $T$ is the temperature, and $m$ and $M$ are the masses of the helium atom and the ion, respectively.

In practice, only the arrival time $t_{A}=t_{D}+t_{0}$ can be measured, with $t_{0}$ denoting the transfer time from the end of the drift tube to the detector. Arrival time distributions are measured for various drift voltages $V$ (typically between $200 \mathrm{~V}$ and $500 \mathrm{~V}$ ) and ion mobility is then obtained from the slope of $t_{A}$ as a function of $V^{-1}$ based on equations (3) and (4) The experimental uncertainty on the absolute value of the CCS is estimated at $2 \%$.

Time resolved fluorescence anisotropy measurements (TRFA). Time resolved anisotropy and fluorescence lifetime was measured on a custom-built set-up. ${ }^{36} \mathrm{~A}$ picosecond laser (PGx03 series Optical Parametric Generators (OPG) from EKSPLA) pumped by a $1 \mathrm{kHz}$ mode-locked laser was used for excitation. The optical design was optimized to produce low divergence at a pulse duration of approximately $20 \mathrm{ps}$. The detection set up is composed of an optical fiber connected to a PMT (R1463, Hamamatsu). The fluorescence decay signal was preamplified by a fast preamplifier (350 MHz Preamplifier SR445A, Stanford Research) and recorded as a function of 
time with a fast digital oscilloscope (Lecroy LT322). The excitations used were set at $500 \mathrm{~nm}$.

The fluorescence lifetimes were obtained using a multi-exponential fitting model on the fluorescence decays given by:

$$
I(t)=\sum_{i} \alpha_{i} e^{-t / \tau_{i}}
$$

where, $\alpha_{i}$ is the amplitude of the decay of the $i^{t h}$ component at time $t$ and $\tau_{i}$ is the lifetime of the $i^{\text {th }}$ component.

For time resolved fluorescence anisotropy measurements, a polarizing beamsplitter cube and a halfwave plate were used to tune the vertical or horizontal linear polarity of the light on the excitation line. A linear broadband polarizer in the range 500 - $720 \mathrm{~nm}$ was used for the collection according to the vertical or horizontal axis. The fluorescence anisotropy decay $r$ was defined as:

$$
r(t)=\frac{I_{V V}(t)-G I_{V H}(t)}{I_{V V}(t)+2 G I_{V H}(t)}
$$

where $I_{V V}$ corresponds to the fluorescence intensity of vertically polarized excitation and vertically polarized emission, $I_{V H}$ to the vertically polarized excitation and horizontally polarized emission and $G$ to the correction factor, reevaluated for each fluorescence anisotropy measurement, with

$$
G=\frac{I_{H V}(t)}{I_{H H}(t)}
$$

where $I_{H V}$ corresponds to the fluorescence intensity of horizontally polarized excitation and vertically polarized emission and $I_{H H}$ to the horizontally polarized excitation and horizontally polarized emission. Anisotropy decays were analyzed with a mono-exponential fitting model using the following equation:

$$
r(t)=r_{0} e^{-t / \theta}
$$


where $r_{0}$ is the anisotropy at time $t=0$ and $\theta$ is the rotational correlation time defined by the Stokes-Einstein relation:

$$
\theta=\frac{\eta V}{k T}
$$

where $\eta$ is the viscosity of the solution, $k$ the Boltzmann constant, $T$ the temperature and $V$ the volume of the rotating object. For a mixture of methanol/glycerol $(10: 90 \%, \mathrm{v} / \mathrm{v}), \eta=253 \mathrm{mPa} . \mathrm{s}$ at $25^{\circ} \mathrm{C}$ and for a mixture of water/glycerol $(10: 90 \%, \mathrm{v} / \mathrm{v}), \eta=165 \mathrm{mPa} . \mathrm{s}$ at $25^{\circ} \mathrm{C} .{ }^{37-38}$ Fluorescence anisotropy decay curves are obtained by averaging five replicates. The quality of the fit in lifetime and anisotropy decay analysis was tested by the chi square value. The hydrodynamic size of clusters was then determined by the radius of a sphere with volume $V$. Error bars are given by the standard error of the mean on the rotational correlation time measured on the six replicates individually.

Steady-state fluorescence, absorption, and excitation. The steady-state fluorescence spectra were recorded by an Ultracompact spectrophotometer Econic (B\&WTek Inc., Newark, DE, U.S.A.) with a resolution of $1.5 \mathrm{~nm}$ via an optical fiber using the same excitation at $500 \mathrm{~nm}$ and appropriate optical filters. A low pass Fast Fourier Transform algorithm was then applied to the spectra in order to smooth the signal. Illumination for absorption measurements was provided by a deuterium-halogen lamp (AvaLight-DH-S-DUV, Avantes). Absorbance was then recorded by a spectrophotometer AvaSpec-2048FT (Avantes). Excitation spectra were obtained using a spectrofluorometer Fluoromax 4 ( Horiba, Jobin Yvon).

\section{RESULTS.}




\section{Nanoclusters in the solid phase}

X-ray Powder Diffraction (XRPD) is used to evaluate the crystal size of nanomaterials based on the Debye-Scherrer equation, as nicely demonstrated by Jin and coworkers for sizing glutathione capped $\mathrm{Au}_{25}(\mathrm{SG})_{18}{ }^{39}$ This routine technique comprises two important structural features: (i) metal core size at wide XR scattering angle, (ii) interparticle packing distance with small angle XR diffraction; the latter being related to the core-ligand shell extension. X-ray powder diffraction analysis was performed for the three powder samples (gold clusters bound to bulky tetrabutyl ammonium cations): $\mathrm{TBA}-\mathrm{Au}_{15}(\mathrm{SG})_{13}, \mathrm{TBA}-\mathrm{Au}_{18}(\mathrm{SG})_{14}$ and $\mathrm{TBA}-\mathrm{Au}_{25}(\mathrm{SG})_{18}$, as shown in fig. 1 .

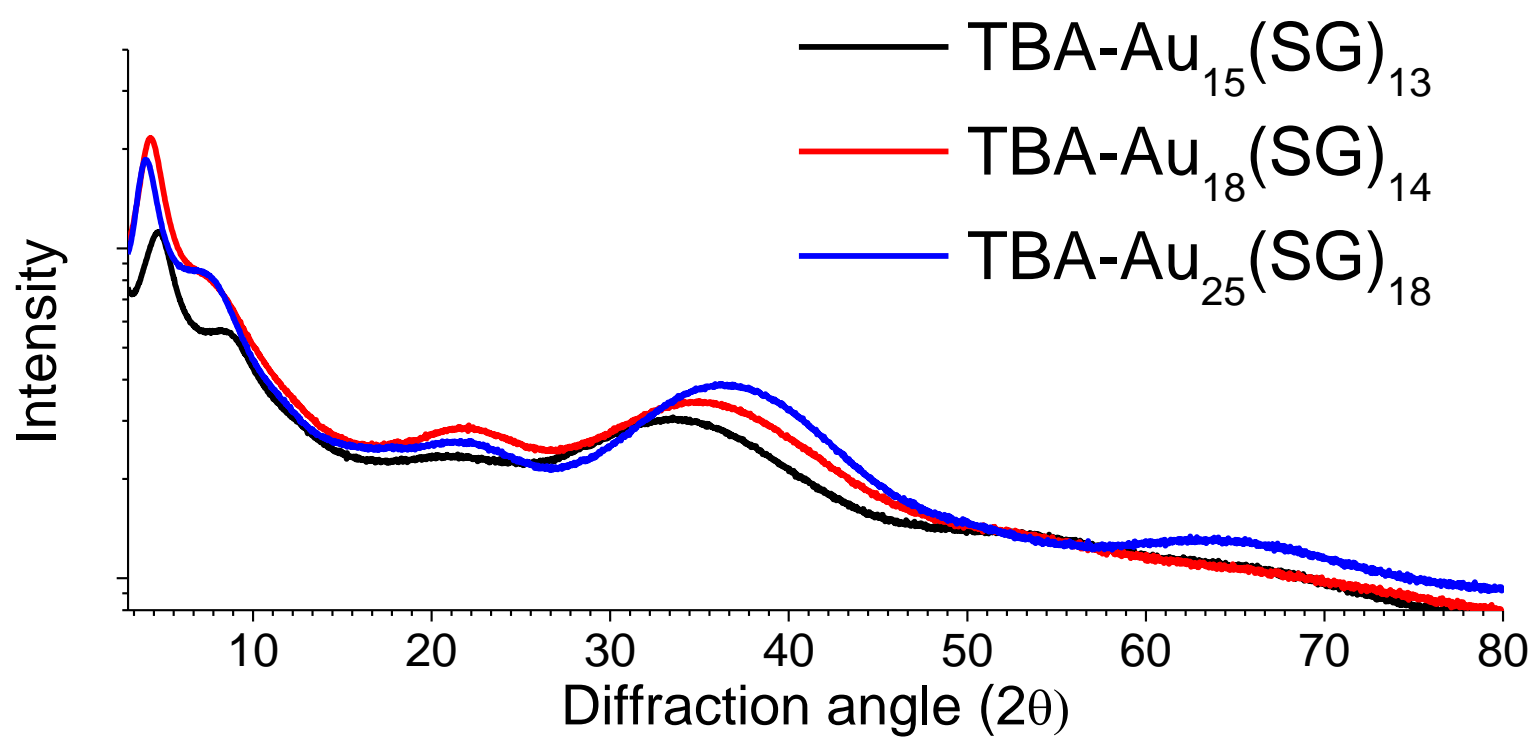

Figure 1: XRD patterns of $\mathrm{Au}_{15}(\mathrm{SG})_{13}, \mathrm{Au}_{18}(\mathrm{SG})_{14}$, and $\mathrm{Au}_{25}(\mathrm{SG})_{18}$ nanoclusters ion-paired with bulky tetrabutyl ammonium cations (TBA).

With wide angle XR diffraction, the powder pattern of $\mathrm{TBA}-\mathrm{Au}_{25}(\mathrm{SG})_{18}$ presents two high peaks at $2 \theta \sim 37^{\circ}$ and $2 \theta \sim 65^{\circ}$ and is very similar to the X-ray powder diffraction analysis reported for $\mathrm{Au}_{25}(\mathrm{SG})_{18 .}{ }^{39}$ At $2 \theta \sim 37^{\circ}$, the shape and the positions of the diffraction peaks are sensitive to the 
number of gold atoms forming the nanoclusters. We applied the Debye-Scherrer equation to the three curves and obtained a gold core diameter of $0.63,0.65$ and $0.73 \mathrm{~nm}$ for $\mathrm{TBA}-\mathrm{Au}_{15}(\mathrm{SG})_{13}$, TBA-Au ${ }_{18}(\mathrm{SG})_{14}$ and TBA-Au $25(\mathrm{SG})_{18}$, respectively. The core diameter TBA-Au 25 is in very good agreement with the estimation from Jin and coworkers for the $\mathrm{Au}_{25}$ core diameter (i.e. $\left.0.74 \mathrm{~nm}\right){ }^{39}$

As expected, bulky tetrabutyl ammonium cations were not found to affect the core diameter and the experimental patterns observed with wide angle XR diffraction are similar between glutathione capped- and TBA ion-paired glutathione capped-gold nanoclusters (see fig. S2 in supporting information, for $\left.\mathrm{Au}_{15}(\mathrm{SG})_{13} \mathrm{NCs}\right)$. The peak with small angle $\mathrm{XR}$ diffraction is linked to the interparticle distance ${ }^{40}$ and can be used to estimate the center-to-center particle distance using Bragg's law. We obtained 1.86, 2.06 and $2.21 \mathrm{~nm}$ for the center-to-center distance in TBA$\mathrm{Au}_{15}(\mathrm{SG})_{13}, \mathrm{TBA}-\mathrm{Au}_{18}(\mathrm{SG})_{14}$ and TBA-Au $25(\mathrm{SG})_{18}$, respectively. The presence of bulky tetrabutyl ammonium cations affects the center-to-center particle distance (due to their steric hindrance), and the experimental patterns observed with small angle XR diffraction are different between glutathione capped- and TBA ion-paired glutathione capped-gold nanoclusters (see fig. S2 in supporting information). A shorter center-to-center particle distance is measured (1.63, 1.65 and $1.79 \mathrm{~nm}$ for $\mathrm{Au}_{15}(\mathrm{SG})_{13}, \mathrm{Au}_{18}(\mathrm{SG})_{14}$ and $\mathrm{Au}_{25}(\mathrm{SG})_{18}$ respectively) for gold clusters without bulky tetrabutyl ammonium cations. If the two layers of ligand shell molecules in the crystal structure are not interpenetrated, then the center-to-center particle distance is roughly the size of the nanocluster (vide infra).

\section{Nanoclusters in the gas phase}

Ion mobility spectrometry allows separating gas phase ions based on the differences of their electrical mobility in a buffer gas. In combination with mass spectrometry (IM-MS) ${ }^{41}$ it is an 
emerging tool for the nanocluster community, and has mainly been used as an additional separation technique to deal with complex mixtures. ${ }^{24}$ The possibility of extracting structural information for metal nanoclusters through collision cross-section measurements has been explored only recently. ${ }^{42}$ In the hard sphere approximation, collision cross-section $\sigma$ and collision radius a (essentially given by the particle radius) are linked by $\sigma=\pi \mathrm{a}^{2} \cdot{ }^{43}$ The examination of the IM-MS map obtained for $\mathrm{Au}_{15}(\mathrm{SG})_{13}$ clusters (fig. 2) first provides information on the structural diversity of this cluster. The extracted arrival time distributions ATDs are monomodal for all the detected charge states of $\mathrm{Au}_{15}(\mathrm{SG})_{13}$, indicating that the corresponding clusters present a single structural type. An important finding in this IM-MS study is that a unique arrival time distribution peak is observed for every nanocluster with different charge states investigated, meaning that the nanocluster presents only a single structure (as shown in Fig. S3 in supporting information). 


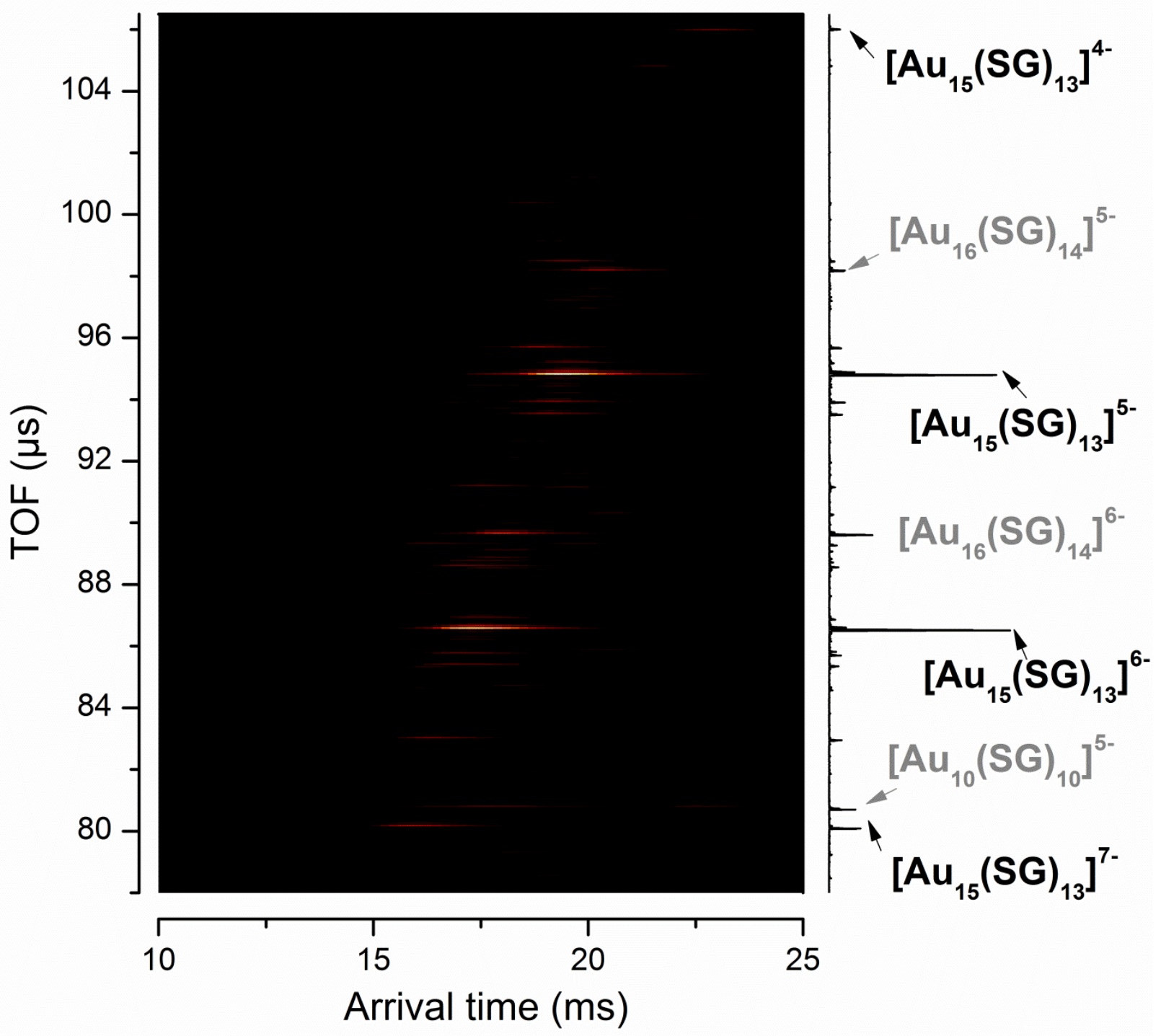

Figure 2: IM-MS map showing time of flight ( $y$ axis) versus arrival drift time ( $x$ axis) for the analysis of $\mathrm{Au}_{15}(\mathrm{SG})_{13}$. The color corresponds to an intensity scale from black (low) to white (high). ATDs extracted for three charge states of $\mathrm{Au}_{15}(\mathrm{SG})_{13}$ are plotted in the inset. The results were obtained using 4.0 Torr Helium as a buffer gas at $298 \mathrm{~K}$, with a $450 \mathrm{~V}$ drift voltage across the drift tube.

As described in the experimental section, an absolute value for the CCS of the clusters can be obtained by measuring ATDs at different drift voltages. The experimental CCSs determined for different charge states for $\mathrm{Au}_{15}(\mathrm{SG})_{13}, \mathrm{Au}_{18}(\mathrm{SG})_{14}$ and $\mathrm{Au}_{25}(\mathrm{SG})_{18}$ nanoclusters are given in Table 
1. For a given cluster, the higher the charge state, the larger the collision cross-section. This may be related to the fact that the ligand molecule is a tripeptide that partially unfolds upon charging in the gas phase, leading to an increase in the collision cross-section. By linking the collision cross section to the cluster radius $\left(\sigma=\pi \mathrm{a}^{2}\right)$, and taking the CCS value interpolated at a charge state equal 0 , as shown in fig. S4 in supporting information (to avoid the "unfolding" effect), the radii are 1.22, 1.31 and $1.47 \mathrm{~nm}$ for $\mathrm{Au}_{15}(\mathrm{SG})_{13}, \mathrm{Au}_{18}(\mathrm{SG})_{14}$ and $\mathrm{Au}_{25}(\mathrm{SG})_{18}$, respectively.

Table 1: Collision cross-section measured in helium at $298 \mathrm{~K}\left({ }^{\mathrm{DT}} \mathrm{CCS} \mathrm{Se}_{\mathrm{He}}\right.$ ) for atomically precise gold nanoclusters as a function of their charge state. Precision on CCS values is $+/-2 \%$.

\begin{tabular}{|c|c|c|c|c|c|}
\hline \multirow[b]{2}{*}{ Au NCs } & \multicolumn{5}{|c|}{$\mathrm{DT}^{\mathrm{DT}} \mathrm{CS}_{\mathrm{He}}\left(\AA^{2}\right)$ vs Charge State } \\
\hline & $4-$ & $5-$ & $6-$ & $7-$ & 8 \\
\hline $\mathrm{Au}_{15}(\mathrm{SG})_{13}$ & 536 & 567 & 604 & 647 & \\
\hline Au18(SG)14 & 565 & 590 & 630 & 662 & 717 \\
\hline $\mathrm{Au}_{25}(\mathrm{SG})_{18}$ & & & 704 & 727 & 770 \\
\hline
\end{tabular}

The effect of adding bulky tetrabutyl ammonium cations on the collision cross-section was evaluated for $\mathrm{Au}_{15}(\mathrm{SG})_{13}$ (see fig. S5 for the 5- charge state). Up to $8 \mathrm{TBA}$, counterion adducts were observed in IM-MS ATDs, for charge state 5- of $\mathrm{Au}_{15}(\mathrm{SG})_{13}$. A linear increase in the total collision cross-section was obtained as a function of the number of TBA adducts (see fig. S5 in supporting information). The collision cross-section was incremented by around $30 \AA^{2}$ for each TBA adduct, leading to a $\sim 40 \%$ increase in the total collision cross-section for the $\left[\mathrm{Au}_{15}(\mathrm{SG})_{13}+\right.$ 8. TBA $]^{5-}$ cluster ions. The corresponding equivalent radius on $\mathrm{Au}_{15} \mathrm{TBA}$ " 0 -charge state" was thus $1.45 \mathrm{~nm}\left(\mathrm{ccs}=667 \AA^{2}\right)$, instead of $1.22 \mathrm{~nm}$ for the " 0 -charge state" $\mathrm{Au}_{15}(\mathrm{SG})_{13}$ clusters. 


\section{Nanoclusters in the liquid phase}

The optical properties of glutathione-protected gold clusters dispersed in solution have been largely explored, in particular with the seminal work of Tsukuda and coworkers. ${ }^{44}$

Photoluminescence (PL) spectra display a broad band in the near IR region centered around 650$700 \mathrm{~nm}$. PL quantum yields are low in aqueous solutions, typically lower than $410^{-3}$. The optical properties of $\mathrm{Au}_{18}(\mathrm{SG})_{14}$ clusters ion-paired with TBA in methanol studied in this work, are presented in Fig. 3. The luminescence intensity was enhanced after pairing with TBA, as can be seen in fig 3a). The intensity increased by nearly factor 4 for $\mathrm{Au}_{18}(\mathrm{SG})_{14}$ clusters ion-paired with TBA in methanol (compared to clusters without ion-pairing in pure water). The fluorescence lifetime of atomically precise $\mathrm{Au}(\mathrm{SG})$ clusters is long and heterogeneous with an average value exceeding $3 \mu \mathrm{s}$. As also reported in a pioneering work on fluorescence anisotropy of BSA protein protected $\mathrm{Au}_{25}$ nanoclusters, ${ }^{28}$ we explored fluorescence lifetimes with polarized excitation. Nanocluster size evaluation can be performed by measuring the rotational diffusion coefficient, which is sensitive to particle size. ${ }^{26} \mathrm{We}$ used a mixture of methanol/glycerol $(10: 90 \%, \mathrm{v} / \mathrm{v})$ in order to slow down $\mathrm{Au}(\mathrm{SG})$ cluster motion in a high viscosity solvent, resulting in measurable anisotropy decay. The anisotropy decay of TBA-Au ${ }_{18}(\mathrm{SG})_{14}$ in methanol/glycerol $(10: 90 \%, \mathrm{v} / \mathrm{v})$ is shown in fig. $3 \mathrm{~b}$ (with excitation at $500 \mathrm{~nm}$ ). The slow decay in anisotropy was fitted to a mono-exponential decay and a recovered correlation time of $987 \mathrm{~ns}$ was obtained for $\mathrm{TBA}-\mathrm{Au}_{18}(\mathrm{SG})_{14}$ (results for other $\mathrm{Au}(\mathrm{SG})$ clusters are given in fig. S6 in supporting information). Using equation (9) given in the Experimental section, the size radius can be extracted from the recovered correlation time. The radii were $1.48,1.57$ and $1.66 \mathrm{~nm}$ for $\mathrm{TBA}-\mathrm{Au}_{15}(\mathrm{SG})_{13}, \mathrm{TBA}-\mathrm{Au}_{18}(\mathrm{SG})_{14}$ and $\mathrm{TBA}-\mathrm{Au}_{25}(\mathrm{SG})_{18}$, respectively. Although fluorescence yields are much lower in water (without adding bulky TBA), we managed to record fluorescence anisotropy decay of gold nanoclusters in water/glycerol 
$(10: 90 \%, v / v)$. The corresponding radii without adding bulky TBA were slightly smaller than that obtained for TBA ion-paired nanoclusters (see Table 2).
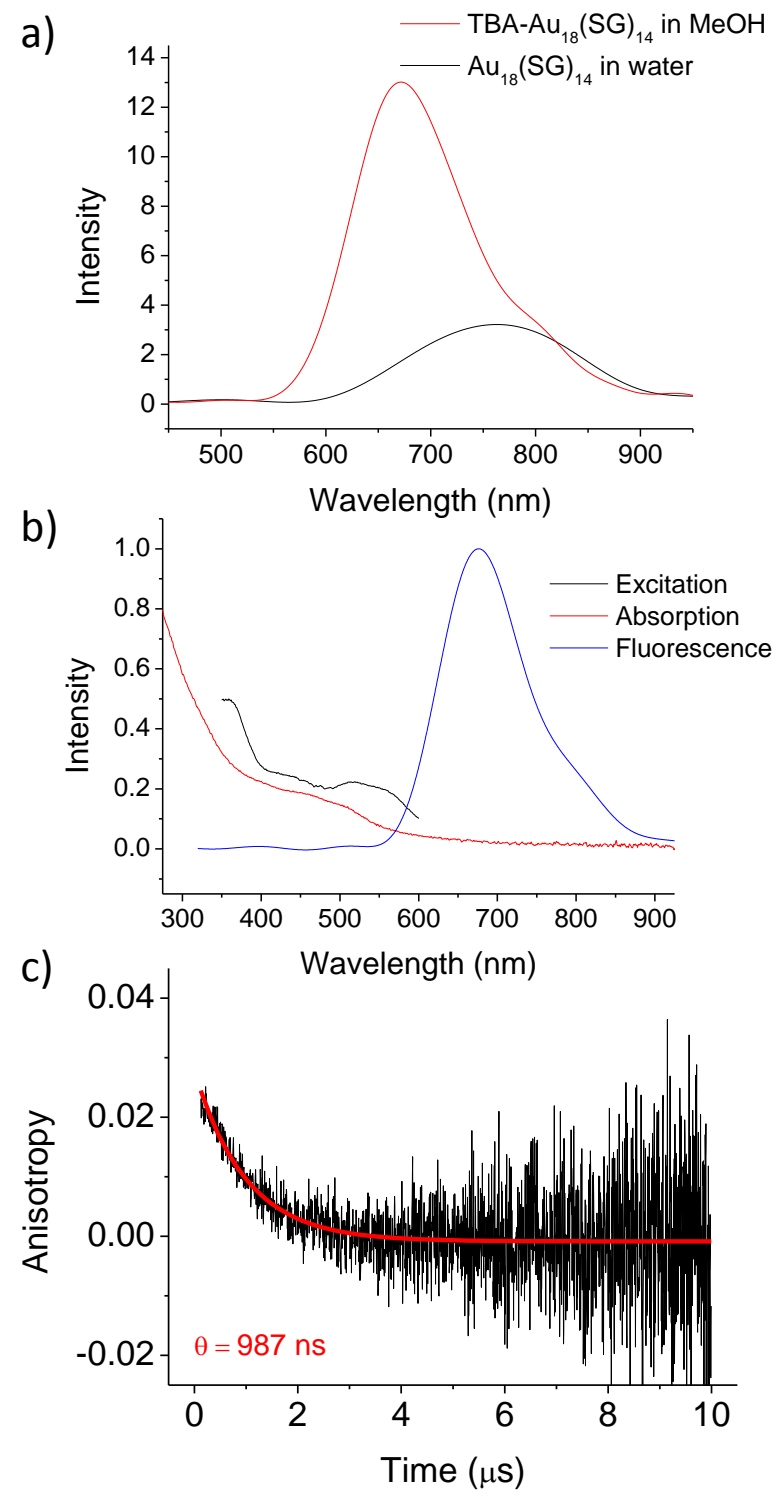

Figure 3: a) Luminescence spectra of $\mathrm{Au}_{18}(\mathrm{SG})_{14}$ in water and TBA-Au ${ }_{18}(\mathrm{SG})_{14}$ in methanol. An absorbance of 0.04 at $500 \mathrm{~nm}$ was measured for both solutions. b) Absorption, photoexcitation (with an emission at $642 \mathrm{~nm}$ ) and emission spectra (with an excitation at $500 \mathrm{~nm}$ ) of $\mathrm{TBA}-\mathrm{Au}_{18}(\mathrm{SG})_{14}$ in methanol. c) Fluorescence anisotropy decay of TBA-Au $18(\mathrm{SG})_{14}$ clusters in methanol/glycerol $(10: 90 \%$, v/v) at $500 \mathrm{~nm}$. A recovered correlation time of 987 ns was obtained from the mono-exponential fitting curve. 


\section{DISCUSSION.}

It was shown that the three techniques investigated, namely X-ray powder diffraction (XRPD), ion mobility mass spectrometry (IM-MS) and time-resolved fluorescence anisotropy (TRFA), can perform sizing for gold nanoclusters in different environments (solid, liquid and gas phases). However, what do these size measurements really mean at this scale? How do these techniques compare with each other and is there any effect of phase on size or size measurements?

X-ray powder diffraction links diffraction angles directly to distances. Interparticle packing distance with small angle XR diffraction (and gold core radius) can be obtained directly. For this technique, there is a low angle cut-off (with the current instrument) of $3^{\circ}$ which correspond to a high limit for interparticle packing distance measurement of $\sim 3 \mathrm{~nm}$. On the other hand, ion mobility mass spectrometry and time-resolved fluorescence anisotropy are associated with the surfaces and volumes of nanoclusters, respectively. To obtain a size, the spherical shape approximation for nanoclusters was used in this work, as it provides a good approximation for compact geometry. This assumption of a quasi-spherical geometry of these clusters may be far from reality for such ultrasmall nanoclusters, and size calculations using geometric and shape considerations could be more accurate. ${ }^{45}$ Concerning accuracy, while size measurement precisions cannot be better than $10 \%$ for XRPD and TRFA, the experimental uncertainties on the CCS values determined were estimated to be no more than $2 \%$. Another advantage of IM-MS is its ease of use as it does not require any sample preparation. Indeed, atomically precise gold nanoclusters can be efficiently placed in the gas phase using the "soft" ionization method of electrospray. For ion mobility mass spectrometry (IM-MS), the limit in IM-MS measurements is mainly given by the limit of the MS instrument to extract cluster size with enough precision. $\sim 1 \mathrm{MDa}$ is a typical high 
mass limit, which correspond to a cluster size of $\sim 5 \mathrm{~nm} .{ }^{46}$ On the other hand, the success of a powder diffraction structural analysis depends on the nature of the sample and of the experimental conditions. The optimal conditions for making a sample "suitable" for diffraction analysis might depend on the solutions used. Concerning the applicability of TRFA, the nanoclusters must obviously be fluorescent. In this work, we propose a strategy to bind NCs to bulky tetraoctylammonium cations to enhance quantum yield luminescence at room temperature. The use of a high viscosity solvent provides measurable anisotropy decay in the size range of 1-3 $\mathrm{nm}$. Further cooling of the solvent temperature could help to further increase the sensitivity of this technique. In the present work, the limitation of this technique is given by the minimum decay time measured with enough precision in anisotropy decay curves which is $\sim 100$ ns, which corresponds to $0.8 \mathrm{~nm}$ for the smallest size. On the other hand, this technique has been used to measure the size of gold-protein clusters for which sizes can reach $\sim 10 \mathrm{~nm} .^{27}$

Both XRPD and IM-MS techniques could gain precision in the structural characterization of nanoclusters by comparing the experimental results (XRPD curve and IM arrival time distributions) with molecular modeling, in particular for the predictions of the structures using a combined global optimization method and DFT calculations. Indeed, XRD curves are sensitive to the exact structures of clusters. ${ }^{34}$ Regarding IM-MS, collision cross-section calculations can be carried out using the trajectory method or the exact hard-sphere scattering model for a calculated structure. ${ }^{47}$ 
Table 2: Summary of size values obtained by XRPD, IM-MS and TRFA techniques for different atomically precise gold nanoclusters.

\begin{tabular}{|c|c|c|c|c|c|c|}
\hline \multicolumn{7}{|c|}{ Cluster size } \\
\hline $\begin{array}{c}\text { Radius } \\
\text { (nm) }\end{array}$ & $\mathrm{Au}_{15}(\mathrm{SG})_{13}$ & $\begin{array}{c}\text { TBA- } \\
\mathrm{Au}_{15}(\mathrm{SG})_{13} \\
\end{array}$ & $\mathrm{Au}_{18}(\mathrm{SG})_{14}$ & $\begin{array}{c}\text { TBA- } \\
\mathrm{Au}_{18}(\mathrm{SG})_{14} \\
\end{array}$ & $\mathrm{Au}_{25}(\mathrm{SG})_{18}$ & $\begin{array}{c}\text { TBA- } \\
\mathrm{Au}_{25}(\mathrm{SG})_{18}\end{array}$ \\
\hline $\begin{array}{c}\text { Solid } \\
\text { phase }^{\text {a) }}\end{array}$ & 0.82 & 0.93 & 0.83 & 1.03 & 0.90 & 1.11 \\
\hline $\begin{array}{c}\text { Gas } \\
\text { phase }^{\text {b) }}\end{array}$ & 1.22 & 1.45 & 1.31 & N/A & 1.47 & N/A \\
\hline $\begin{array}{l}\text { Liquid } \\
\left.\text { phase }^{\mathrm{c}}\right)\end{array}$ & 1.45 & 1.48 & 1.50 & 1.57 & 1.66 & 1.66 \\
\hline
\end{tabular}

One of the primary goals of this work was to compare the size measurements carried out using solid, liquid and gas phase methods for selected glutathione-protected gold clusters. Table 2 gives the following order for a given nanocluster: $\mathrm{NC}$ size $\mathrm{s}_{\text {solid phase }}<\mathrm{NC}$ size $\mathrm{gas}_{\text {phase }}<\mathrm{NC}$ size $\mathrm{e}_{\text {liquid phase. }}$ In the solid phase, the NC size is extracted from the center-to-center particle distance. This size measurement in the solid phase, using the XRPD technique may underestimate the real size of NCs, since there are two layers of glutathione peptide molecules that can interpenetrate each other, as can be observed in the crystal structure. In the liquid phase, the NC size is extracted for the rotational diffusion of the fluorescent NCs. In the liquid medium, a thin electric dipole layer of the solvent adheres to the nanocluster surface. This layer influences the motion of the particle in the medium, leading to a hydrodynamic size measurement that slightly overestimates the size of the bare NC. Of note, TBA ion-paired and non-ion-paired nanoclusters show almost same sizes in liquid whereas the case in solid is totally different. In solid phase, the presence of bulky tetrabutyl ammonium cations leads to a larger center-to-center particle distance. This is certainly due to their steric hindrance preventing an efficient interpenetration of ligands. In liquid phase, a measure of 
"hydrodynamic" size of clusters is performed, and includes a solvation shell. This solvation shell erases the prominent effect of counterions. ${ }^{48}$

Finally, IM-MS measurements were performed on multiply charged NCs and the charge was found to have an effect on the collision cross-section. An interpolation procedure was proposed to remove the charge effect on the collision cross-section measurements, as it may affect the accuracy of this measurement. The choice of other ligands (that cannot unfold upon charging) for atomically precise gold cluster size measurements would limit this effect.

\section{CONCLUSIONS.}

In this letter, we aimed to compare solid, liquid and gas phase methods used to measure the size of selected glutathione-protected gold clusters. We evaluated how these different measurements can be interpreted to give consistent results. X-ray powder diffraction (XRPD), ion mobility mass spectrometry (IM-MS) and time-resolved fluorescence anisotropy (TRFA) can be used to obtain sample size measurements in the size range of 1-3 nm for gold nanoclusters in different environments (solid, liquid and gas phases), relatively straightforwardly. We believe that these techniques can be a useful addition to the set of characterization tools available for nanoclusters in the size range of 1-3 nm. While absolute values differ, as discussed above, relative values as a function of size are in good agreement, and the relative values obtained in the three phases are consistent.

It is clear that for nanoscience and technology to be effective, they must be underpinned by dimensional nanometrology. The present work points out the limit of size definition for monolayer-protected gold nanoclusters, due to the subtle interplay between environmental effects 
and intrinsic technical accuracy. In this size range, it is more a matter of defining accurate metrics (collision cross-sections, rotational diffusion coefficients, etc.) than really measuring size.

\section{ASSOCIATED CONTENT}

Supporting Information. ESI mass spectra of the as-synthesized gold NCs. XRD patterns of $\mathrm{Au}_{15}$ nanoclusters. Arrival time distributions extracted from the IM-MS map for $\mathrm{Au}_{15} \mathrm{NCs}$. Collision cross-section measured for gold NCs. ESI mass spectrum and collision cross-section values for $\mathrm{Au}_{15} \mathrm{NCs}$ as a function of TBA number. Fluorescence anisotropy decay curves of gold NCs in methanol/glycerol $(10: 90 \%, \mathrm{v} / \mathrm{v})$. The following files are available free of charge.

\section{AUTHOR INFORMATION}

\section{Corresponding Author}

*rodolphe.antoine@univ-lyon1.fr

\section{ACKNOWLEDGMENT}

The research leading to these results received partial funding from the European Research Council under the European Union's Seventh Framework Programme (FP7/2007-2013 grant agreement no. 320659).

\section{REFERENCES}

1. Smith, D. J., Chapter 1 Characterization of Nanomaterials Using Transmission Electron Microscopy. In Nanocharacterisation (2), The Royal Society of Chemistry: 2015; pp 1-29.

2. Lupini, A. R.; Rashkeev, S. N.; Varela, M.; Borisevich, A. Y.; Oxley, M. P.; van Benthem, K.; Peng, Y.; de Jonge, N.; Veith, G. M.; Pantelides, et al. Chapter 2 Scanning Transmission Electron Microscopy. In Nanocharacterisation; The Royal Society of Chemistry, 2007, pp 28-65. 3. Jin, R., Quantum Sized, Thiolate-Protected Gold Nanoclusters. Nanoscale 2010, 2, 343362.

4. Roduner, E., Size Matters: Why Nanomaterials Are Different. Chem. Soc. Rev. 2006, 35, 583-592. 
5. Lu, Y.; Chen, W., Sub-Nanometre Sized Metal Clusters: From Synthetic Challenges to the Unique Property Discoveries. Chem. Soc. Rev. 2012, 41, 3594-3623.

6. Jin, R.; Zeng, C.; Zhou, M.; Chen, Y., Atomically Precise Colloidal Metal Nanoclusters and Nanoparticles: Fundamentals and Opportunities. Chem. Rev. 2016, 116, 10346-10413.

7. Chakraborty, I.; Pradeep, T., Atomically Precise Clusters of Noble Metals: Emerging Link between Atoms and Nanoparticles. Chem. Rev. 2017, 117, 8208-8271.

8. Tsukuda, T.; Häkkinen, H., Introduction. Frontiers of Nanoscience. Elsevier, Amsterdam, 2015, 9, 1-7.

9. Zhu, M.; Aikens, C. M.; Hollander, F. J.; Schatz, G. C.; Jin, R., Correlating the Crystal Structure of a Thiol-Protected Au25 Cluster and Optical Properties. J. Am. Chem. Soc. 2008, 130, 5883-5885.

10. Parker, J. F.; Fields-Zinna, C. A.; Murray, R. W., The Story of a Monodisperse Gold Nanoparticle: Au25L18. Acc. Chem. Res. 2010, 43, 1289-1296.

11. Heaven, M. W.; Dass, A.; White, P. S.; Holt, K. M.; Murray, R. W., Crystal Structure of the Gold Nanoparticle [N(C8H17)4][Au25(SCH2CH2ph)18]. J. Am. Chem. Soc. 2008, 130, 37543755.

12. Jadzinsky, P. D.; Calero, G.; Ackerson, C. J.; Bushnell, D. A.; Kornberg, R. D., Structure of a Thiol Monolayer-Protected Gold Nanoparticle at $1.1 \AA$ Resolution. Science 2007, 318, 430433.

13. Levi-Kalisman, Y.; Jadzinsky, P. D.; Kalisman, N.; Tsunoyama, H.; Tsukuda, T.; Bushnell, D. A.; Kornberg, R. D., Synthesis and Characterization of Au102(P-MBA)44 Nanoparticles. J. Am. Chem. Soc. 2011, 133, 2976-2982.

14. Canton, P.; Fazzini, P. F.; Meneghini, C.; Benedetti, A.; Pozzi, G., Chapter 6 - Nanoscale Characterization of Metal Nanoclusters by Means of X-Ray Diffraction (XRD) and Transmission Electron Microscopy (TEM) Techniques A2 - Corain, B. In Metal Nanoclusters in Catalysis and Materials Science, Schmid, G.; Toshima, N., Eds. Elsevier: Amsterdam, 2008; pp 129-147.

15. Salorinne, K.; Lahtinen, T.; Koivisto, J.; Kalenius, E.; Nissinen, M.; Pettersson, M.; Häkkinen, H., Nondestructive Size Determination of Thiol-Stabilized Gold Nanoclusters in Solution by Diffusion Ordered NMR Spectroscopy. Anal. Chem. 2013, 85, 3489-3492.

16. Carney, R.; Young Kim, J.; Qian, H.; Jin, R.; Mehenni, H.; Stellacci, F.; Bakr, O., Determination of Nanoparticle Size Distribution Together with Density or Molecular Weight by 2D Analytical Ultracentrifugation, Nat. Commun. 2011, 2, 335.

17. Plascencia-Villa, G.; Demeler, B.; Whetten, R. L.; Griffith, W. P.; Alvarez, M.; Black, D. M.; José-Yacamán, M., Analytical Characterization of Size-Dependent Properties of Larger Aqueous Gold Nanoclusters. J. Phys. Chem. C 2016, 120, 8950-8958.

18. Kimura, K.; Sugimoto, N.; Sato, S.; Yao, H.; Negishi, Y.; Tsukuda, T., Size Determination of Gold Clusters by Polyacrylamide Gel Electrophoresis in a Large Cluster Region. J. Phys. Chem. C 2009, 113, 14076-14082.

19. Azubel, M.; Koivisto, J.; Malola, S.; Bushnell, D.; Hura, G. L.; Koh, A. L.; Tsunoyama, H.; Tsukuda, T.; Pettersson, M.; Häkkinen, H. et al., Electron Microscopy of Gold Nanoparticles at Atomic Resolution. Science 2014, 345, 909-912.

20. Vergara, S.; Lukes, D. A.; Martynowycz, M. W.; Santiago, U.; Plascencia-Villa, G.; Weiss, S. C.; de la Cruz, M. J.; Black, D. M.; Alvarez, M. M.; Lopez-Lozano, X.; et al., MicroED Structure of Au146(PMBA)57 at Subatomic Resolution Reveals a Twinned FCC Cluster. J. Phys. Chem. Lett. 2017, 8, 5523-5530. 
21. Harkness, K. M.; Cliffel, D. E.; McLean, J. A., Characterization of Thiolate-Protected Gold Nanoparticles by Mass Spectrometry. Analyst 2010, 135, 868-874.

22. Lu, Y.; Chen, W., Application of Mass Spectrometry in the Synthesis and Characterization of Metal Nanoclusters. Anal. Chem. 2015, 87, 10659-10667.

23. Hamouda, R.; Bertorelle, F.; Rayane, D.; Antoine, R.; Broyer, M.; Dugourd, P., Glutathione Capped Gold Aun(SG)m Clusters Studied by Isotope-Resolved Mass Spectrometry. Int. J. Mass Spectrom. 2013, 335, 1-6.

24. Baksi, A.; Ghosh, A.; Mudedla, S. K.; Chakraborty, P.; Bhat, S.; Mondal, B.; Krishnadas, K. R.; Subramanian, V.; Pradeep, T., Isomerism in Monolayer Protected Silver Cluster Ions: An Ion Mobility-Mass Spectrometry Approach. J. Phys. Chem. C 2017, 121, 13421-13427.

25. Angel, L. A.; Majors, L. T.; Dharmaratne, A. C.; Dass, A., Ion Mobility Mass Spectrometry of Au25(SCH2CH2PH)18 Nanoclusters. ACS Nano 2010, 4, 4691-4700.

26. Hayashi, T.; Ishizaki, Y.; Michihata, M.; Takaya, Y.; Tanaka, S.-I., Nanoparticle Sizing Method Based on Fluorescence Anisotropy Analysis. Measur. 2015, 59, 382-388.

27. Li, C.; Sutter, J. U.; Birch, D. J. S.; Chen, Y. Fluorescence Anisotropy of Protein - Gold Nanoclusters, 2012 12th IEEE International Conference on Nanotechnology (IEEE-NANO), 2023 Aug. 2012; 2012; pp 1-4.

28. Raut, S.; Chib, R.; Rich, R.; Shumilov, D.; Gryczynski, Z.; Gryczynski, I., Polarization Properties of Fluorescent BSA Protected Au25 Nanoclusters. Nanoscale 2013, 5, 3441-3446.

29. Qu, X.; Li, Y.; Li, L.; Wang, Y.; Liang, J.; Liang, J., Fluorescent Gold Nanoclusters: Synthesis and Recent Biological Application. J. Nanomat. 2015, 2015, 23.

30. Chen, L.-Y.; Wang, C.-W.; Yuan, Z.; Chang, H.-T., Fluorescent Gold Nanoclusters: Recent Advances in Sensing and Imaging. Anal. Chem. 2015, 87, 216-229.

31. Wu, Z.; Jin, R., On the Ligand's Role in the Fluorescence of Gold Nanoclusters. Nano Lett. 2010, 10, 2568-2573.

32. Pyo, K.; Thanthirige, V. D.; Kwak, K.; Pandurangan, P.; Ramakrishna, G.; Lee, D., Ultrabright Luminescence from Gold Nanoclusters: Rigidifying the Au(I)-Thiolate Shell. J. Am. Chem. Soc. 2015, 137, 8244-8250.

33. Russier-Antoine, I.; Bertorelle, F.; Vojkovic, M.; Rayane, D.; Salmon, E.; Jonin, C.; Dugourd, P.; Antoine, R.; Brevet, P.-F., Non-Linear Optical Properties of Gold Quantum Clusters. The Smaller the Better. Nanoscale 2014, 6, 13572-13578.

34. Bertorelle, F.; Russier-Antoine, I.; Calin, N.; Comby-Zerbino, C.; Bensalah-Ledoux, A.; Guy, S.; Dugourd, P.; Brevet, P.-F.; Sanader, Ž.; Krstić, M.; et al., Au10(SG)10: A Chiral Gold Catenane Nanocluster with Zero Confined Electrons. Optical Properties and First-Principles Theoretical Analysis. J. Phys. Chem. Lett. 2017, 8, 1979-1985.

35. Simon, A.-L.; Chirot, F.; Choi, C. M.; Clavier, C.; Barbaire, M.; Maurelli, J.; Dagany, X.; MacAleese, L.; Dugourd, P., Tandem Ion Mobility Spectrometry Coupled to Laser Excitation. Rev. Sci. Instrum. 2015, 86, 094101.

36. Soleilhac, A.; Bertorelle, F.; Dugourd, P.; Girod, M.; Antoine, R., Monitoring MethanolInduced Protein Unfolding by Fluorescence Anisotropy Measurements of Covalently Labelled Rhodamine Probe. Eur. Phys. J. D 2017, 71, 142.

37. Afshar Ghotli, R.; Abdul Aziz, A. R.; Atadashi, I. M.; Hasan, D. B.; Kong, P. S.; Aroua, M. K., Selected Physical Properties of Binary Mixtures of Crude Glycerol and Methanol at Various Temperatures. J. Ind. Eng. Chem. 2015, 21, 1039-1043.

38. Takamura, K.; Fischer, H.; Morrow, N. R., Physical Properties of Aqueous Glycerol Solutions. J. Petrol. Sci. Eng. 2012, 98, 50-60. 
39. Wu, Z.; Chen, J.; Jin, R., One-Pot Synthesis of Au25(SG)18 2- and 4-Nm Gold Nanoparticles and Comparison of Their Size-Dependent Properties. Adv. Funct. Mater. 2011, 21, 177-183.

40. Lavenn, C.; Albrieux, F.; Bergeret, G.; Chiriac, R.; Delichere, P.; Tuel, A.; Demessence, A., Functionalized Gold Magic Clusters: Au25(SphNH2)17. Nanoscale 2012, 4, 7334-7337.

41. Harkness, K. M.; Balinski, A.; McLean, J. A.; Cliffel, D. E., Nanoscale Phase Segregation of Mixed Thiolates on Gold Nanoparticles. Angew. Chem. Int. Ed. 2011, 50, 10554-10559.

42. Ligare, M. R.; Baker, E. S.; Laskin, J.; Johnson, G. E., Ligand Induced Structural Isomerism in Phosphine Coordinated Gold Clusters Revealed by Ion Mobility Mass Spectrometry. Chem. Commun. 2017, 53, 7389-7392.

43. Wyttenbach, T.; Bleiholder, C.; Bowers, M. T., Factors Contributing to the Collision Cross Section of Polyatomic Ions in the Kilodalton to Gigadalton Range: Application to Ion Mobility Measurements. Anal. Chem. 2013, 85, 2191-2199.

44. Negishi, Y.; Nobusada, K.; Tsukuda, T., Glutathione-Protected Gold Clusters Revisited: Bridging the Gap between Gold(I)-Thiolate Complexes and Thiolate-Protected Gold Nanocrystals. J. Am. Chem. Soc. 2005, 127, 5261-5270.

45. Mori, T.; Hegmann, T., Determining the Composition of Gold Nanoparticles: A Compilation of Shapes, Sizes, and Calculations Using Geometric Considerations. J. Nano. Res. 2016, 18, 295.

46. Yuan, X.; Yao, Q.; Yu, Y.; Luo, Z.; Xie, J., Chapter 6 Novel Synthetic Strategies for Thiolate-Protected Au and Ag Nanoclusters: Towards Atomic Precision and Strong Luminescence. In Functional Nanometer-Sized Clusters of Transition Metals: Synthesis, Properties and Applications, The Royal Society of Chemistry: 2014; pp 131-168.

47. Shvartsburg, A. A.; Jarrold, M. F., An Exact Hard-Spheres Scattering Model for the Mobilities of Polyatomic Ions. Chem. Phys. Lett. 1996, 261, 86-91.

48. Bertorelle, F.; Moulin, C.; Soleilhac, A.; Comby-Zerbino, C.; Dugourd, P.; RussierAntoine, I.; Brevet, P.-F.; Antoine, R., Bulky Counterions: Enhancing the Two-Photon Excited Fluorescence of Gold Nanoclusters. ChemPhysChem, 2017, 10.1002/cphc.201701186. 
TOC Graphic.

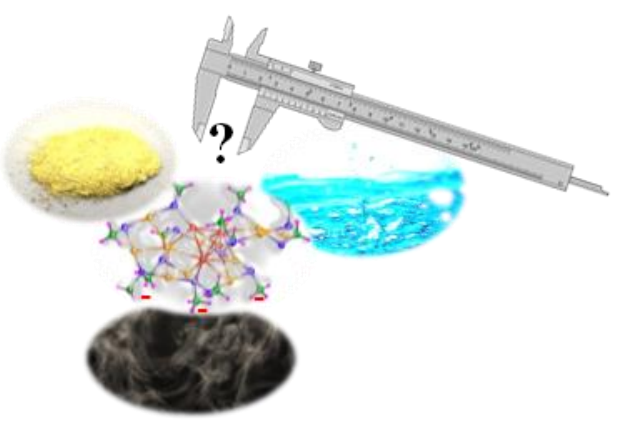

